\title{
DIAGNÓSTICO DE CUERPOS EXTRAÑOS GASTROINTESTINALES EN CANINOS MEDIANTE ECOGRAFÍA Y RADIOLOGÍA
}

\author{
Diagnosis of Gastrointestinal Foreign Bodies in the Canine Through \\ ULTRASONOGRAPHY AND RADIOLOGY
}

Jacqueline Cahua U. ${ }^{1}$ y Diego Díaz C. ${ }^{2}$

\section{Resumen}

\begin{abstract}
El objetivo de este trabajo fue determinar si la técnica de imagen diagnóstica radiográfica o la ecográfica es la que mejor apoya al diagnóstico de un probable cuerpo extraño gastrointestinal. Se consideraron 13 casos de caninos con diagnóstico presuntivo de obstrucción gastrointestinal. Los pacientes fueron sometidos paralelamente a pruebas radiológicas y ecográficas. Ecográficamente, se observó la presencia de cuerpos extraños en los 13 casos, mientras radiográficamente se identificaron 3 cuerpos extraños y 7 fueron sospechosos de obstrucción por cuerpo extraño, debido a la sobre distensión intestinal. Los cuerpos extraños fueron identificados ecográficamente por la sombra acústica distal y grados variables de reflexión que producen en la superficie. Además, ecográficamente, se pudo observar engrosamiento de la pared gástrica e intestinal en algunos animales, y líquido libre peritoneal en un paciente. Los hallazgos encontrados en este trabajo sugieren que en animales pequeños con una probable obstrucción gastrointestinal, la ecografía podría ser usada para determinar el diagnóstico y puede ser más apropiada que la radiología.
\end{abstract}

Palabras clave: cuerpo extraño, gastrointestinal, radiografía, radiología, ultrasonido

\section{Abstract}

The aim of this study was to determine whether the technique of ultrasound or radiographic image diagnosis, better supports a probable diagnosis of gastrointestinal foreign body. Thirteen dogs with presumptive diagnosis of gastrointestinal obstruction were subjected to radiological tests and ultrasound evaluation. Ultrasonography showed the presence of foreign bodies in all 13 cases, while through radiology, three foreign bodies were identified and seven were suspected of foreign body obstruction due to the intestinal distension. The foreign bodies were sonographically identified by the distal acoustic shadow and various degrees of reflection produced on the surface. In addition, the thickening of the stomach and intestinal wall in some animals, and free peritoneal fluid in a patient could be observed through ultrasound images. The findings in this study suggest that ultrasound can be used for the diagnosis of gastrointestinal foreign

${ }^{1}$ Clínica de Animales Menores, ${ }^{2}$ Laboratorio de Farmacología, Facultad de Medicina Veterinaria, Universidad Nacional Mayor de San Marcos, Lima 
bodies in small animals with a likely gastrointestinal obstruction, and may be more appropriate than radiology.

Key words: foreign body, gastrointestinal, radiography, radiology, ultrasound

\section{INTRODUCCIÓN}

La ingestión de cuerpos extraños en el canino puede producir una obstrucción total o parcial del tracto gastrointestinal. La técnica diagnóstica más frecuentemente utilizada en animales pequeños ante la sospecha de cuerpos extraños gastrointestinales es la radiografía. Los cuerpos extraños radiopacos pueden ser identificados mediante radiografías simples, aunque los signos radiológicos pueden variar con el grado, duración y localización de la obstrucción (Riedesel, 2003). No obstante, hay otras causas de obstrucción del tracto gastrointestinal, tales como la intususcepción, neoplasias y torsiones intestinales, que producen signos radiográficos similares (Riedesel, 2003). Por otro lado, la ecografía es ampliamente usada para el estudio de imagen del tracto gastrointestinal en humanos sospechosos de tener cuerpos extraños (Spina et al., 2000).

Existe un limitado número de reportes documentando el diagnóstico ecográfico de cuerpos extraños gastrointestinales en mascotas (Tidwell y Penninck, 1992; Hoffmann, 2003; Hunt et al., 2004; Tyrrell y Beck, 2006), por lo que el objetivo del presente estudio fue evaluar las imágenes radiográficas y ecográficas como técnicas diagnósticas de probables obstrucciones gastrointestinales por cuerpo extraño en el canino.

\section{Materiales y Métodos}

El estudio se llevó a cabo en la Clínica de Animales Menores de la Facultad de
Medicina Veterinaria de la Universidad Nacional Mayor de San Marcos, Lima. Se trabajó con 13 pacientes caninos que llegaron a la clínica en un lapso de seis meses con diagnóstico presuntivo de obstrucción gastrointestinal por cuerpo extraño (1 Scotish Terrier, 3 Pitbull, 1 Bullterrier, 1 Siberian Husky, 1 Braco Alemán, 2 Golden Retriever, 1 Perro Peruano sin Pelo, 1 cruce de Labrador y 1 mestizo). La edad de los canes varió entre tres meses a siete años (promedio: 2.6 años) y los signos clínicos se manifestaron entre dos días a dos semanas, aunque mayormente fueron de 2 a 3 días. El vómito fue un signo común, pero, además, se observó inapetencia (11/13). Sólo en tres canes se encontró una masa abdominal palpable.

Los pacientes fueron sometidos a pruebas radiográficas simples y ecográficas. Para el estudio radiográfico se tomaron placas abdominales en posición decúbito lateral. En las radiografías se buscaron cambios radiológicos consistentes que sugieran la presencia de cuerpos extraños gastrointestinales, como distensión gástrica, y sobre distensión del intestino delgado. Se considera distención gástrica cuando el fondo gástrico es mayor que el ancho de tres espacios intercostales (Tyrrell y Beck, 2006) y sobre distención intestinal cuando el cociente del máximo diámetro del intestino delgado y la altura del cuerpo de la lumbar 5 en su punto más estrecho es inferior a 1.6 (Riedesel, 2003). El examen ecográfico abdominal completo se hizo con un ecógrafo SA 600V Medison y un transductor convexo de $3.5 \mathrm{MHz}$. Los cuerpos extraños fueron reconocidos por su forma, por la fuerte sombra acústica distal y grados variables de reflexión de la superficie (Riedesel, 2003). 
Cuadro 1. Hallazgos ecográficos en 13 perros con diagnóstico presuntivo de obstrucción gastrointestinal por cuerpo extraño

\begin{tabular}{lc}
\hline Hallazgo ecográfico & $\mathrm{N}^{\circ}$ de observaciones \\
\hline Distensión gástrica & 4 \\
Sobre distensión de intestino delgado & 8 \\
Distensión de colon & 2 \\
Fluido peritoneal libre & 1 \\
Cuerpo extraño & 13 \\
Fluido en estómago & 8 \\
Fluido en intestino delgado & 12 \\
Engrosamiento de pared gástrica & 5 \\
Engrosamiento en pared de intestino delgado & 4 \\
Cuerpo extraño en intestino delgado & 10 \\
Cuerpo extraño en estómago & 4 \\
Cuerpo extraño en colon descendente & 1 \\
\hline
\end{tabular}

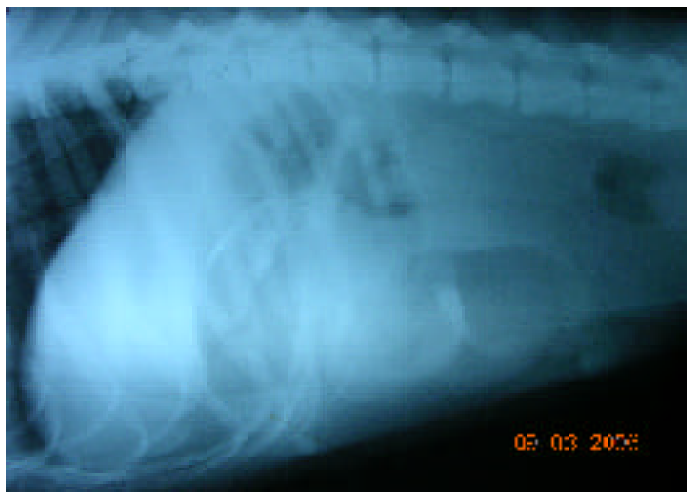

(a)

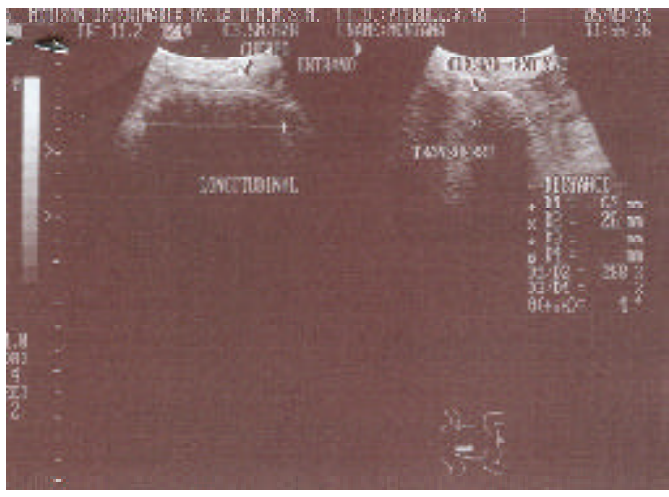

(b)

Figura 1. Imagen radiológica (a) de un canino en posición de decúbito lateral donde se observa la dilatación intestinal por gases. En la imagen ecográfica del mismo paciente (b) se observa un cuerpo extraño de $67 \mathrm{~mm}$ de longitud y $26 \mathrm{~mm}$ de diámetro transverso, de forma tubular, localizado en el intestino. En la intervención quirúrgica se extrajo una coronta de choclo.

\section{Resultados}

En el examen radiográfico se pudo detectar un cuerpo extraño en solo tres anima- les $(23 \%)$. Otros hallazgos radiográficos fueron acumulación excesiva de gas (10), sobre distensión intestinal (8), sospecha de obstrucción por presencia de gas (7) y distención gástrica (1). 
En el examen ecográfico se detectó los cuerpos extraños en los 13 animales (100\%) (Cuadro 1). El cuerpo extraño fue removido quirúrgicamente en 11 canes, donde nueve se localizaron en el intestino delgado y dos en el estómago. Un cuerpo extraño fue retirado por endoscopía del estómago y el cuerpo extraño en el colon descendente fue eliminado por el ano, luego de un tránsito baritado. Los cuerpos extraños extraídos correspondieron a corontas de choclo (8), pepas de durazno (2), trapos (2), pepa de mango (1), panty de nylon (1), palito (1) y un trozo de plástico. Algunos perros tenían más de un cuerpo extraño.

\section{Discusión}

Radiográficamente es dificil observar los cuerpos extraños cuando están compuestos por materia orgánica y, más aun, si existe liquido ascítico; por otro lado, ecográficamente es difícil evidenciar los cuerpos extraños cuando son muy pequeños o no producen retención de líquido intestinal intraluminal, y en casos de meteorismo marcado que impide la llegada del ultrasonido hasta el cuerpo extraño. Así mismo, no se puede diferenciar un cuerpo extraño del material fecal que produce sombra acústica cuando los primeros están en el colon.

En el presente estudio, la ultrasonografía identificó la presencia de cuerpo extraño en los 13 animales, mientras que el estudio radiográfico pudo evidenciarlo en solo tres animales, y en otros siete se tuvo la sospecha por la colección de gases intestinales. Estos hallazgos son semejantes a otros reportes (Tidwell y Penninck, 1992; Tyrrell y Beck, 2006). Así mismo, el estudio radiográfico identificó ocho animales con sobre distención de intestino delgado, lo que es un signo de obstrucción intestinal, aunque no necesariamente originado por un cuerpo extraño (Papazoglou et al., 2003; Riedesel, 2003). Esta sobre distensión del intestino delgado se observó también en el examen ecográfico.
Otros hallazgos ecográficos adicionales incluyeron engrosamiento de la pared gástrica, que se observó en cinco animales, de los cuales uno presentaba el cuerpo extraño a nivel de intestino delgado y otro a nivel de colon descendente. En casos de neoplasias se observa una pérdida de definición de las capas de la pared (Riedesel, 2003; Penninck, 2004). Según el estudio de Penninck et al. (1998), en la neoplasia gástrica hay un engrosamiento de 1.0-2.7 cm. En el presente estudio, los tres casos de engrosamiento de la pared intestinal fueron probablemente debido a un deterioro vascular en el sitio del cuerpo extraño como resultado de una severa inflamación y alteración de la pared intestinal (Tyrrell y Beck, 2006). Otro hallazgo ecográfico fue fluido peritoneal en un paciente con cuerpo extraño a nivel de colon. El mecanismo del desarrollo del fluido peritoneal es desconocido.

El estudio radiográfico es la prueba de imagen usualmente usada cuando se sospecha de cuerpo extraño gastrointestinal, en tanto que la ecografía es usada como examen de ayuda cuando el resultado radiográfico no es definitivo (Hoffman, 2003). Sería más económico si solo se usara una prueba para el diagnóstico. Radiográficamente es difícil observar los cuerpos extraños cuando están compuestos por materia orgánica, y mucho menos si existe líquido ascítico. Ecográficamente es dificil evidenciar los cuerpos extraños cuando son muy pequeños, o no producen retención de líquido intestinal intraluminal, y en casos de meteorismo marcado que impide la llegada del ultrasonido hasta el cuerpo extraño. Asimismo, no se puede diferenciar un cuerpo extraño del material fecal que produce sombra acústica cuando se encuentra en el colon.

Los resultados de este estudio son similares a los encontrados en estudios semejantes, donde la ecografía detectó la presencia de cuerpos extraños gastrointestinales que no fueron visualizados en la radiografía simple (Tidwell y Penninck, 1992; Tyrrell y Beck, 2006). 
Los perros con cuerpos extraños gastrointestinales pueden tener una variedad de signos no específicos como vómitos, deshidratación, dolor abdominal (Riedesel, 2003), que dependen de la localización y grado de obstrucción y la alteración del flujo sanguíneo intestinal (Strombeck, 1995). Los líquidos y gases retenidos producen una sobre distensión luminal que puede observarse radiográficamente y que será mayor cuando la obstrucción es más completa y más distal (Riedesel, 2003). En el curso inicial de la obstrucción hay mayor cantidad de gas que de líquidos (Fig. 1) (Tyrrell y Beck, 2006), pero con el transcurrir del tiempo aumenta la secreción y el secuestro de fluidos, dando como resultado un aumento del volumen de fluido intraluminal (Strombeck, 1995). En obstrucciones parciales, el intestino delgado puede no estar distendido, generando cambios menos obvios en la radiografía simple, sobre todo si son recientes (Riedesel, 2003).

La dilatación de las asas intestinales no es un signo patognomónico de la obstrucción por cuerpo extraño. Masas intra y extramurales pueden comprimir los intestinos y causar una obstrucción mecánica. El íleo funcional (parálisis) ocurre por anomalías vasculares o neuromusculares en la pared intestinal (Riedesel, 2003) que resulta en una acumulación anormal de fluidos y gas intestinal. Por otro lado, los cuerpos extraños radiopacos pueden ser visualizados en radiografías simples, pero aquellos no radiopacos o los que son opacados por ingesta son difíciles de detectar. En estos casos puede estar indicado un estudio de contraste, aunque con ciertas limitaciones, pues está contraindicado cuando se sospecha de una perforación intestinal o está contemplada una cirugía inmediata (Riedesel, 2003).

Se puede concluir que la ecografía es un estudio de imagen que da mayor probabilidad de encontrar un cuerpo extraño gastrointestinal que la radiografía simple, por lo menos, con objetos de materia orgánica que suelen ser radiolúcidos. Además, la ecografía aporta información adicional como la calidad de pared gastrointestinal y la presencia de líquido ascítico.

\section{Literatura Citada}

1. Hoffman KL. 2003. Sonographic sign of gastroduodenal linear foreign body in 3 dogs. Vet Radiol Ultrasound 44: 466469.

2. Hunt GB, Worth A, Marchevsky A. 2004. Migration of wooden skewer foreign body from the gastrointestinal tract in eight dogs. J Small Anim Pract 45: 362-366.

3. Papazoglou LG, Patsikas MN, Rallis T. 2003. Intestinal foreign bodies in dog and cats. Compend Contin Educ Pract Vet 25: 830-843.

4. Penninck DG. 2004. Tracto gastrointestinal. En: Nyland T, Matton J. (eds). Ecografía en pequeños animales. $2^{\mathrm{da}} \mathrm{ed}$. Barcelona, España: Ed. Gráfica Multimédica.

5. Penninck DG, Moore AS, Gliatto J. 1998. Ultrasonography of canine gastric epithelial neoplasia. Vet Radiol Ultrasound 39: 342-346.

6. Riedesel EA. 2003. El intestino delgado. En: D. Thrall (ed). Manual de diagnóstico radiológico veterinario. Cap. 48. $4^{\text {ta }}$ ed. España: Ed. Elsevier. p 639-656.

7. Spina P, Minniti S, Bragheri R. 2000. Usefulness of ultrasonography in gastric foreign body retention. Pediatr Radiol 30 : 840-841.

8. Strombeck D. 1995. Enfermedades digestivas de los animales pequeños. $2^{\text {la }}$ ed. Buenos Aires, Argentina: Interamericana. $796 \mathrm{p}$.

9. Tidwell AS, Penninck DG. 1992. Ultrasonography of gastrointestinal foreign body. Vet Radiol Ultrasound 33: 160-169.

10. Tyrrell D, Beck C. 2006. Survey of the use of radiography vs. ultrasonography in the investigation of gastrointestinal foreign body in small animals. Vet Radiol Ultrasound 47: 404-408. 